Алгебра и анализ

Том. 15 (2003), вып. 3
St. Petersburg Math. J.

Vol. 15 (2004), No. 3, Pages 449-468 S 1061-0022(04)00817-9

Article electronically published on March 31, 2004

\title{
INTEGRALS RELATED TO THE CANTOR FUNCTION
}

\author{
E. A. GORIN AND B. N. KUKUSHKIN
}

\begin{abstract}
The classical Cantor function is included in a continuous one-parameter family. Each function in this family takes Lebesgue measure to a measure supported on the set of dyadic rationals on $(0,1)$. Analytic properties of the Fourier and Mellin transforms of these measures in dependence on the parameter value are studied.
\end{abstract}

\section{INTRODUCTION}

Usually, seeking primitives of elementary functions is a rather dull job (however, this rule has some exceptions: see, e.g., [1]). Often the primitive is not an elementary function or, even if it is, it either occurs in all $\mathrm{ABC}$ books or can be obtained by integration by parts from primitives known universally. It is more interesting (and useful) to evaluate integrals that depend on a parameter, and complex analysis methods introduce much loveliness in this task. However, in some situations one deals with the integrals of classical but nonelementary functions, and complex analysis methods seem to be irrelevant. As is clear from the title, we are going to consider such a setting. It should be noted that, still, a central role in what follows will be played by the invocation of holomorphic functions.

For some accidental reasons, one of the authors arrived at the idea to evaluate the integral of the square of the classical Cantor monotone function (staircase) over the interval $[0,1]$. By the way, afterwards, a "poll" showed that nobody was surprised by the mere possibility of a precise evaluation, but none of the respondents could say what the resulting number would be (it is $3 / 10$ ).

Everything discussed in the sequel is dictated by intrinsic logic: first, it becomes interesting to evaluate the integrals of other natural powers, then the question arises about the asymptotics as $n \rightarrow \infty$, and this leads spontaneously to the integrals of complex powers and to incorporation of the Cantor function into a certain one-parameter family.

We note that the integrals of all natural powers of the classical Cantor function are rationals; the list of them begins with

$$
\frac{1}{2}, \frac{3}{10}, \frac{1}{5}, \frac{33}{230}, \frac{5}{46}, \frac{75}{874}, \frac{611}{8740}, \frac{97653}{1673710} .
$$

As has already been said, we incorporate the Cantor monotone function into a oneparameter family $\left\{C_{q}\right\}, q \in(2, \infty)$. The classical Cantor function corresponds to $q=3$. We study the behavior of the integrals

$$
I_{q}(\lambda)=\int_{0}^{1} C_{q}(t)^{\lambda} d t \quad \text { and } \quad E_{q}(\lambda)=\int_{0}^{1} e^{\lambda C_{q}(t)} d t .
$$

The definition of the Cantor function will be recalled below. However, the results can be described in terms of discrete measures, and this will be done immediately.

2000 Mathematics Subject Classification. Primary 26A30.

Key words and phrases. Cantor function, Fourier transform, Mellin transform. 
Let $Q_{2}(0,1)$ denote the set of all dyadic rationals on the interval $(0,1)$. Every number $x \in Q_{2}(0,1)$ admits a unique representation in the form $m / 2^{n}$, where $m$ and $n$ are natural numbers and $m$ is odd. The number $n$ will be called the rank of $x$ and will be denoted by $\operatorname{rank}(x)$. There are precisely $2^{n-1}$ numbers of rank $n$. Now, we introduce a probability measure $\mu_{q}$ by putting

$$
\mu_{q}(\{x\})=(q-2) / q^{n} \quad \text { if } \operatorname{rank}(x)=n
$$

Up to notation, the integrals indicated above are classical integral transforms of $\mu_{q}$ :

$$
I_{q}(\lambda)=\int_{0}^{1} x^{\lambda} \mu_{q}(d x) \quad \text { and } \quad E_{q}(\lambda)=\int_{0}^{1} e^{\lambda x} \mu_{q}(d x) .
$$

Though in this case we deal with sums, we use the symbol of the (Lebesgue) integral intentionally, and moreover, in the form accepted in probability theory. Strictly speaking, it would then be more consistent to indicate the domain of integration as a subscript, but we avoid doing so (for aesthetic reasons, which are justified by the fact that $\mu_{q}$ has no mass at the endpoints of $[0,1])$.

We list the main results. We shall give some formulas that make it possible to find $I_{q}(n)$ recursively for natural $n$, and also we shall express $I_{q}(n)$ in terms of Bernoulli numbers. These formulas give some idea about the arithmetic nature of the numbers $I_{q}(n)$, but they are hardly applicable to the study of the asymptotics of these quantities as $n \rightarrow \infty$. It turns out that, as $\lambda \rightarrow+\infty$, we have the two-sided estimate

$$
c_{1} \lambda^{-\alpha} \leq I_{q}(\lambda) \leq c_{2} \lambda^{-\alpha}
$$

where $\alpha=\log _{2} q$, but no power-like asymptotics is available. However, the picture is simpler and more interesting than it could have been expected:

$$
I_{q}(\lambda)=\lambda^{-\alpha} \Phi_{q}\left(\log _{2} \lambda\right)+O\left(\lambda^{-\alpha-1}\right)
$$

where $\Phi_{q}$ is a function analytic in the strip $|\operatorname{Im} s|<\pi / 2 \log 2$, and $\Phi_{q}(s+1)=\Phi_{q}(s)$. Hereinafter, $\log x$ stands for the natural logarithm if $x>0$.

Next, the function $I_{q}(\lambda)$ extends to a function meromorphic in $\mathbb{C}$ and having simple poles at the points $-\alpha-2 \pi i n / \log 2, n \in \mathbb{Z}(\mathbb{Z}$ is the group of integers). The residues at these points admit simple expressions in terms of the Riemann $\zeta$-function. Clearly, $E_{q}(\lambda)$ extends to an entire function. It turns out that there exists $q_{0} \in(2,3)$ such that for $q<q_{0}$ this entire function has infinitely many pure imaginary zeros, and for $q>q_{0}$ (in particular, for the classical Cantor function) it has none.

Our arguments are based on the fact that the Cantor functions satisfy simple linear functional equations. The latter lead to functional equations for the entire functions $E_{q}(\lambda)$, and this launches a powerful machinery. Surely, such an approach is applicable in a far more general setting, but we have chosen to maintain the equilibrium between generality and simplicity, especially because it is still not clear who in the world would need all this stuff, however attractive our calculations could seem.

The first author (in the alphabetical order) has reported these results not only in private conversations, but also in official talks, but the proofs have not been published before. As yet, nobody has "caught us in plagiarism", though we shall not be surprised if we learn that something similar has occurred in the literature.

B. M. Makarov has read the first version of this paper. We have taken his suggestions into account (in particular, the last two sections have been rewritten entirely), and one of his remarks (about the Fourier coefficients of $\Phi_{q}$ ) is formulated as a theorem. 


\section{$\S 1$. Cantor Functions}

On the set of finite segments (closed intervals) $[a, b] \subset \mathbb{R}$, where $\mathbb{R}$ is the field of reals, we define the operation

$$
\operatorname{Del}_{q}([a, b]) \stackrel{\text { def }}{=}[a, b] \backslash(a+(b-a) / q, b-(b-a) / q) .
$$

Clearly, this operation converts a segment into a pair of disjoint segments (we recall that $q>2)$.

In a natural way, this operation extends to the collection of finite unions of mutually disjoint segments. Then this "cutting procedure" can be iterated. This enables us to define the Cantor set $S_{q}$ for every $q>2$ in the following way:

$$
S_{q} \stackrel{\text { def }}{=} \bigcap_{n=1}^{\infty} \operatorname{Del}_{q}^{n}([0,1])
$$

In particular, $S_{3}$ is the classical Cantor ternary set.

It is easily seen that meas $\left(S_{q}\right)=0$, where $\operatorname{meas}(S)$ is the Lebesgue measure of a set $S \subset \mathbb{R}$; however, the Hausdorff dimension of $S_{q}$ is $1 / \log _{2} q$, that is, this metric characteristic distinguishes the sets $S_{q}$. For a fixed $q$, we put $\alpha=\log _{2} q$. In what follows, unless otherwise is stated explicitly, $\alpha$ always denotes this number. The intervals constituting the complement of $S_{q}$ (contiguous intervals) are enumerated in a natural way by the numbers $x \in Q_{2}(0,1)$; moreover, the mutual position (rightwards, leftwards) of the intervals is the same as that of the corresponding numbers. In the terminology of the classical function theory, these sets are similar. The rank of a contiguous interval is defined to be the rank of its number. Thus, an interval of rank $n$ is eliminated at the $n$th step of the construction of a Cantor set. The lengths (Lebesgue measures) of all $2^{n-1}$ intervals of rank $n$ coincide and are equal to $(q-2) / q^{n}$.

The Cantor function $C_{q}$ is a continuous function on $[0,1]$ that takes the value $x \in$ $Q_{2}(0,1)$ everywhere on the interval with number $x$. This is a fairly bizarre edifice. The functions $C_{2}(t)=t$ and $C_{\infty}(t)=1 / 2,0<t<1$, are obtained as pointwise limits.

Let $x \in Q_{2}(0,1)$, and let $\operatorname{rank}(x)=n$. Then

$$
x=2^{-n}\left(1+2 \varepsilon_{1}+2^{2} \varepsilon_{2}+\cdots+2^{n-1} \varepsilon_{n-1}\right),
$$

where $\varepsilon_{k}^{2}=\varepsilon_{k}$. For the corresponding contiguous interval $\left(a_{x}, b_{x}\right)$ we have

$$
b_{x}=(q-1) q^{-n}\left(1+q \varepsilon_{1}+q^{2} \varepsilon_{2}+\cdots+q^{n-1} \varepsilon_{n-1}\right) .
$$

In particular,

$$
\left.b_{x}+b_{1-x}=1+(q-2) / q^{n} \quad \text { (i.e. } a_{x}+b_{1-x}=1\right) \quad \text { and } \quad q b_{x / 2}=b_{x} .
$$

The following statement is a direct consequence of these formulas.

Lemma 1. For the Cantor function, we have

$$
C_{q}(t)+C_{q}(1-t)=1 \quad \text { and } \quad C_{q}(t)=2 C_{q}(t / q) .
$$

Lemma 2. For every $t \in[0,1]$, we have

$$
t /(q-1) \leq C_{q}(t)^{\alpha} \leq t .
$$

These two inequalities are sharp, and the function $C_{q}(t)^{\alpha} / t$ has no limit as $t \rightarrow 0$.

Proof. For instance, we prove the left inequality. It suffices to check the inequality for the right endpoint $b$ of a contiguous interval, i.e., to show that $(b /(q-1))^{1 / \alpha} \leq C_{q}(b)$. 
But since $1 / \alpha<1$, it follows that

$$
\begin{aligned}
(b /(q-1))^{1 / \alpha} & =\left(q^{-n}\left(1+q \varepsilon_{1}+q^{2} \varepsilon_{2}+\cdots\right)\right)^{1 / \alpha} \\
& =2^{-n}\left(1+q \varepsilon_{1}+q^{2} \varepsilon_{2}+\cdots\right)^{1 / \alpha} \\
& \leq 2^{-n}\left(1+2 \varepsilon_{1}+2^{2} \varepsilon_{2}+\cdots\right) \\
& =C_{q}(b) .
\end{aligned}
$$

Equality is attained at the endpoints of the leftmost contiguous intervals of each rank.

Under the mapping $C_{q}$, the contiguous intervals (together with their endpoints) are taken to dyadic rationals, and the Cantor set without the endpoints is taken bijectively onto the complement of the dyadic rationals. On the level of measures, the induced mapping takes Lebesgue measure to the measure $\mu_{q}$ defined before. So, for every bounded Borel function $f$ we have

$$
\int_{0}^{1}\left(f \circ C_{q}\right)(t) d t=\int_{0}^{1} f(x) \mu_{q}(d x) .
$$

In particular $\left(f(x)=x^{\lambda}\right.$ or $\left.f(x)=e^{\lambda x}\right)$, the two definitions of the functions $I_{q}$ and $E_{q}$ match.

As usual, the measure $\mu_{q}$ is related to a distribution function $m_{q}$ by the formula

$$
\mu_{q}([x, y))=m_{q}(y)-m_{q}(x) \text { for every half-open interval }[x, y) \text {; }
$$

we normalize $m_{q}$ by the condition $m_{q}(0)=0$. This function is left continuous and has jumps of size $m_{q}(\{x\})$ only at the points $x \in Q_{2}(0,1)$. By using $\mu_{q}$, we can rewrite the integrals as Lebesgue-Stieltjes integrals.

Lemma 1 implies that

$$
m_{q}(x+0)+m_{q}(1-x)=1 \quad \text { and } \quad m_{q}(x)=q m_{q}(x / 2) ;
$$

Lemma 2 implies that

$$
x^{\alpha} \leq m_{q}(x) \leq m_{q}(x+0) \leq(q-1) x^{\alpha} .
$$

Conversely, these relations imply the formulas in the lemmas. In particular, $x^{-\alpha} m_{q}(x)$ has no limit as $x \rightarrow 0$.

\section{§2. Elementary inequalities}

There is a simple relationship between the behavior of $I_{q}$ and $E_{q}$. Below we use the obvious formula $I_{q}(n)=E_{q}^{(n)}(0)$ for natural $n$. The following lemma shows that the functions $I_{q}(\lambda)$ and $E_{q}(-\lambda)$ have similar behavior as $\lambda \rightarrow+\infty$. We observe that $E_{q}(\lambda)=e^{\lambda} E_{q}(-\lambda)$ (this follows from Lemma 1 ).

Lemma 3. As $\lambda \rightarrow+\infty$, we have

$$
0 \leq E_{q}(-\lambda)-I_{q}(\lambda)=O\left(\lambda^{-\alpha-1}\right) .
$$

Proof. The difference in question is nonnegative because $1-x \leq e^{-x}$ on the real line. Since $\lambda>0$, we have $I_{q}(\lambda+1)<I_{q}(\lambda)$. Integration by parts yields

$$
\begin{gathered}
E_{q}(-\lambda)-I_{q}(\lambda+1)=\int_{0}^{1}\left[e^{\lambda x}-(1-x) \lambda+1\right] d m_{q}(x) \\
=e^{-\lambda}+\int_{0}^{1}\left[\lambda e^{-\lambda x}-(\lambda+1)(1-x)^{\lambda}\right] m_{q}(x) d x .
\end{gathered}
$$


The rightmost term becomes greater if we replace the coefficient of $e^{-\lambda x}$ by $\lambda+1$. This makes the integrand nonnegative, so that the substitution of $(q-1) x^{\alpha}$ for $m_{q}(x)$ does not reduce the integral. Next,

$$
\int_{0}^{1} x^{\alpha} e^{-\lambda x} d x-\int_{0}^{1} x^{\alpha}(1-x)^{\lambda} d x<\Gamma(\alpha+1) / \lambda^{\alpha+1}-B(\alpha+1, \lambda+1) .
$$

If we use the well-known relationship between Euler integrals, we finish the proof with the help of the asymptotic formula

$$
\lambda^{\beta} \Gamma(\lambda) / \Gamma(\lambda+\beta)=1-\beta(\beta-1) / 2 \lambda+O\left(\lambda^{-2}\right)
$$

(see, e.g., 2]), which is a consequence of the "Stirling series".

Lemma 4. We have

$$
\varliminf_{\lambda \rightarrow+\infty} \lambda^{\alpha} E_{q}(-\lambda)>0 \text { and } \varlimsup_{\lambda \rightarrow+\infty} \lambda^{\alpha} E_{q}(-\lambda)<\infty,
$$

but the function $\lambda^{\alpha} E_{q}(-\lambda)$ has no limit at infinity. The same is true concerning $I_{q}(\lambda)$.

Proof. Both inequalities follow from the estimates for $m_{q}$. By Lemma 3, it suffices to prove the inequalities and the absence of the limit for the function $\lambda^{\alpha} E_{q}(-\lambda)$. Should the limit exist, by Karamata's Tauberian theorem (see, e.g., [3]), the limit of $x^{-\alpha} m_{q}(x)$ as $x \rightarrow 0$ would also exist. However, it has already been noted that this function has no limit as $x \rightarrow 0$.

More complete information about the behavior of these functions on the positive semiaxis will be given later. The absence of the limit in Lemma 4 looks like a subtle statement: the proof requires the "anatomy" of the Cantor function and a Tauberian theorem. In the sequel it will be shown that it is possible to argue entirely on the basis of certain identities that follow from the functional equations.

It is useful to remember the following simple limit objects:

$$
\begin{aligned}
I_{2}(\lambda) & =1 /(\lambda+1), & E_{2}(\lambda) & =\left(e^{\lambda}-1\right) / \lambda, \\
I_{\infty}(\lambda) & =1 / 2^{\lambda}, & E_{\infty}(\lambda) & =e^{\lambda / 2} .
\end{aligned}
$$

\section{§3. THE PRINCIPAL IDENTITIES}

Lemma 1 yields simple relations for $E_{q}$ and $I_{q}$. We observe that $I_{q}$ extends to a function analytic in the half-plane $\operatorname{Re} \lambda>-\alpha$ (this follows from Lemma 2).

Theorem 1. For $\operatorname{Re} \lambda>-\alpha$, the function $I_{q}$ obeys the formula

$$
\left(q \cdot 2^{\lambda}-1\right) I_{q}(\lambda)=q-2+\int_{0}^{1}\left(1+C_{q}(t)\right)^{\lambda} d t
$$

and for all $\lambda \in \mathbb{C}$ the function $E_{q}$ obeys the formula

$$
q \cdot E_{q}(2 \lambda)=(q-2) \cdot e^{\lambda}+\left(e^{\lambda}+1\right) E_{q}(\lambda) .
$$

Proof. In both cases the matter is quite easy. First, we split the domain of integration (the segment $[0,1]$ ) into three parts: the first contiguous interval and the two complementary segments. On the contiguous interval, the integrand is constant. By using Lemma 1 , we reduce the integrals over the complementary segments to the initial integral, after which it remains to simplify the expression. For instance,

$$
q \cdot \int_{1-1 / q}^{1} e^{2 \lambda C_{q}(t)} d t=q \cdot e^{2 \lambda} \int_{0}^{1 / q} e^{-2 \lambda C_{q}(t)} d t=e^{2 \lambda} E_{q}(-\lambda)=e^{\lambda} E_{q}(\lambda) .
$$


Remark. A functional equation for the Laplace transform

$$
E_{q}(\lambda, z)=\int_{0}^{1} e^{\lambda C_{q}(t)+z t} d t
$$

can be deduced in a similar way; however, that equation is not so simple as this one.

Assuming that $-\pi<\arg \lambda<\pi$, we put $\lambda^{\rho}=\exp \rho \log \lambda$. Let

$$
F_{\rho, q}(\lambda)=\lambda^{\rho} E_{q}(\lambda) /\left(e^{\lambda}-1\right) .
$$

Then formula (2) implies the relation

$$
\left(q / 2^{\rho}\right) F_{\rho, q}(2 \lambda)=(q-2) \lambda^{\rho} e^{\lambda} /\left(e^{2 \lambda}-1\right)+F_{\rho, q}(\lambda) .
$$

For what follows, two particular cases are important: $\rho=1$ and $\rho=\alpha$. In these cases we shall write $F_{1}$ and $F_{q}$, respectively (though it is equally logical to write $F_{\alpha}$ in the latter case). Clearly, $F_{1}$ depends on $q$. On the positive semiaxis, these functions are bounded.

\section{§4. Calculation of the integral $I_{q}(n)$}

For $\lambda$ equal to a natural number $n$, we can expand the integrand in formula (1), obtaining the recursive relation

$$
\left(q \cdot 2^{n}-1\right) I_{q}(n)=q-2+\sum_{k=0}^{n}\left(\begin{array}{l}
n \\
k
\end{array}\right) I_{q}(k) .
$$

In the integral formula for $I_{q}$ we can replace $C_{q}$ with $1-C_{q}$; this yields two more recursive relations:

$$
\left(q \cdot 2^{n}-2\right) I_{q}(n)=q-2+2 \sum_{k \leq n}^{\prime}\left(\begin{array}{l}
n \\
k
\end{array}\right) I_{q}(k)
$$

and

$$
q \cdot 2^{n} I_{q}(n)=q-2+2 \sum_{k \leq n}^{\prime \prime}\left(\begin{array}{l}
n \\
k
\end{array}\right) I_{q}(k),
$$

where summation is over the odd $k$ in the first case and over the even $k$ in the second. Now, the obvious identity $I_{q}(1)=1 / 2$ can easily be supplemented with the following:

$$
\begin{aligned}
I_{q}(2) & =\frac{q}{2(2 q-1)}, \\
I_{q}(3) & =\frac{q+1}{4(2 q-1)}, \\
I_{q}(4) & =\frac{q(2 q+5)}{2(2 q-1)(8 q-1)}, \\
I_{q}(5) & =\frac{q^{2}+5 q+1}{2(2 q-1)(8 q-1)} .
\end{aligned}
$$

For $n \geq 2$ we put

$$
\chi_{n}(q)=(2 q-1)(8 q-1) \cdots\left(2 \cdot 4^{m} q-1\right), \quad \text { where } m=[n / 2]-1 .
$$

Let

$$
\psi_{n}(q)= \begin{cases}2 \chi_{n}(q) & \text { if } n \text { is even } \\ 2^{n-1} \chi_{n}(q) & \text { if } n \text { is odd. }\end{cases}
$$

From (4) and (5) we deduce by induction that $I_{q}(n)=\varphi_{n}(q) / \psi_{n}(q)$, where the $\varphi_{n}$ are polynomials of degree $[n / 2]$ and with integral coefficients. 
We need some elementary facts about the Bernoulli numbers (see, e.g., [2] for the details; clearly, many other references can be given, but in the handbook [2] the reader will find a concise presentation with complete proofs).

The Bernoulli polynomials $B_{n}(t)$ are defined by the relation

$$
\frac{\lambda e^{t \lambda}}{e^{\lambda}-1}=\sum_{n=0}^{\infty} B_{n}(t) \frac{\lambda^{n}}{n !} \quad(|\lambda|<2 \pi) .
$$

The numbers $B_{n}=B_{n}(0)$ are called the Bernoulli numbers. It is easily seen that $B_{0}=$ $1, B_{1}=-1 / 2$, and that $B_{n}=0$ for odd $n>1$. The subsequent numbers are

$$
\frac{1}{6}, \quad-\frac{1}{30}, \quad \frac{1}{42}, \quad-\frac{1}{30}, \quad \frac{5}{66},-\frac{691}{2730}, \quad \frac{7}{6}
$$

(the last number is $B_{14}$ ), and afterwards the numerators grow rapidly. Among numerous identities known here, we only need the following, which can easily be verified:

$$
B_{n}(1 / 2)=-\left(1-2^{1-n}\right) B_{n}
$$

Applying (3) with $\rho=1$ and (7), we deduce that

$$
\left(2^{n-1} q-1\right) F_{1}^{(n)}(0)=-(q-2)\left(2^{n-1}-1\right) B_{n} .
$$

In other words, for $|\lambda|<2 \pi$ we have

$$
F_{1}(\lambda)=1-(q-2) \sum_{n}^{\prime \prime} \frac{2^{n-1}-1}{q \cdot 2^{n-1}-1} \cdot \frac{B_{n}}{n !} \cdot \lambda^{n},
$$

where the sum with primes means that summation is over even positive $n$.

The Taylor coefficients of $E_{q}$ can be expressed in a fairly easy way in terms of the Taylor coefficients of $F_{1}$. Since $I_{q}(n)=E_{q}^{(n)}(0)$ for natural $n$, by formula (8) we obtain the following supplement to the recursive relations.

Theorem 2. For all natural $n \geq 2$, we have

$$
I_{q}(n)=\frac{1}{n+1}-(q-2) \sum_{k \leq n}^{\prime \prime}\left(\begin{array}{l}
n \\
k
\end{array}\right) \frac{2^{k-1}-1}{q \cdot 2^{k-1}-1} \cdot \frac{B_{k}}{n-k+1},
$$

where the primes mean that summation is over even positive $k$.

From (8) it follows that for $|\lambda|<2 \pi$ the function $q \mapsto F_{1}(\lambda)$ admits analytic continuation in the parameter $q$ to the complex plane without the points $q=1 /\left(2 \cdot 4^{m}\right.$ ) (at these points, the function has poles), and without the limit point $q=0$. The relationship between $F_{1}$ and $E_{q}$ shows that the same is true for $E_{q}$. Furthermore, by Theorem 1 the condition $|\lambda|<2 \pi$ can be lifted for $E_{q}$. Surely and quite naturally, the zeros of the polynomials (6) lie precisely at the singularities of $E_{q}$ as a function of $q$.

\section{§5. Asymptotics on the ReAl axis}

For $\rho=\alpha$, formula (3) is particularly simple:

$$
F_{q}(2 \lambda)=f_{q}(\lambda)+F_{q}(\lambda)
$$

where

$$
f_{q}(\lambda)=(q-2) \lambda^{\alpha} e^{\lambda} /\left(e^{2 \lambda}-1\right) .
$$

Therefore,

$$
F_{q}(\lambda)=\sum_{k=1}^{\infty} f_{q}\left(\lambda / 2^{k}\right)
$$


if $\operatorname{Re} \lambda>0$. For such $\lambda$, we put

$$
G_{q}(\lambda)=\sum_{k=0}^{\infty} f_{q}\left(2^{k} \lambda\right) \quad \text { and } \quad H_{q}(\lambda)=F_{q}(\lambda)+G_{q}(\lambda) .
$$

The functions $F_{q}, G_{q}$, and $H_{q}$ are analytic in the half-plane $\operatorname{Re} \lambda>0$; moreover, $H_{q}(2 \lambda)=$ $H_{q}(\lambda)$. Let

$$
R_{q}(\lambda)=G_{q}(\lambda)+e^{-\lambda} F_{q}(\lambda)
$$

Then for $\lambda>0$ the function $R_{q}(\lambda)$ is bounded and positive, and $R_{q}(\lambda)=O\left(\lambda^{\alpha} e^{-\lambda}\right)$ as $\lambda \rightarrow+\infty$.

It is easily seen that

$$
\lambda^{\alpha} E_{q}(-\lambda)=H_{q}(\lambda)-R_{q}(\lambda) .
$$

Finally, for $s \in \mathbb{R}$ we put $\Phi_{q}(s)=H_{q}\left(2^{s}\right)$. Clearly, $\Phi_{q}$ extends to a function analytic in the strip $|\operatorname{Im} s|<\pi /(2 \log 2)$, and $\Phi_{q}(s+1)=\Phi_{q}(s)$. In particular, formula (9) implies the following statement.

Theorem 3. We have

$$
\lambda^{\alpha} E_{q}(-\lambda)=\Phi_{q}\left(\log _{2} \lambda\right)+O\left(\lambda^{\alpha} e^{-\lambda}\right)
$$

as $\lambda \rightarrow+\infty$.

Combined with Lemma 3, Theorem 3 yields the asymptotics of the integrals $I_{q}(\lambda)$ as $\lambda \rightarrow+\infty$ :

$$
\lambda^{\alpha} I_{q}(\lambda)=\Phi_{q}\left(\log _{2} \lambda\right)+O\left(\lambda^{-\alpha-1}\right) .
$$

The remainder term in Theorem 3 is a negative and rapidly decaying function. Thus, for $\lambda>0$ we have

$$
\lambda^{\alpha} I_{q}(\lambda)<\lambda^{\alpha} E_{q}(-\lambda)<\Phi_{q}\left(\log _{2} \lambda\right) ;
$$

however,

$$
\varlimsup_{\lambda \rightarrow+\infty} \lambda^{\alpha} I_{q}(\lambda)=\max _{0 \leq s \leq 1} \Phi_{q}(s) .
$$

Clearly,

$$
\varliminf_{\lambda \rightarrow+\infty} \lambda^{\alpha} I_{q}(\lambda)=\min _{0 \leq s \leq 1} \Phi_{q}(s) .
$$

Moreover, if $\lambda>0$ and $n$ is natural, then the following limit exists:

$$
\lim _{n \rightarrow \infty} q^{n} I_{q}\left(2^{n} \lambda\right)=\lambda^{-\alpha} H_{q}(\lambda) .
$$

For large natural $n$, the sequence $n^{\alpha} I_{q}(n)$ is approximated well by the sequence $\Phi_{q}\left(\left\{\log _{2} n\right\}\right)$, where $\{x\}$ is the fractional part of $x$. It is well known (see, e.g., 4 Chapter 3]) that the sequence $\left\{\log _{2} n\right\}$ is highly irregular; for example, its subsequence corresponding to $n=3^{r}$ is uniformly distributed on $[0,1]$. However, for small values of $q$

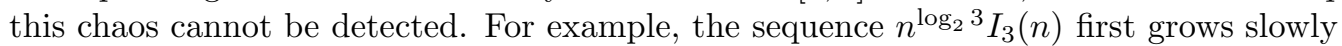
but monotonically, remaining smaller than 2, and apparently the value $n=500$ is still very far from the point of the first maximum. By our request, E. V. Idzikovskil designed a computer program for calculating the values of $\Phi_{3}(s)$. It has turned out that

$$
1.99661 \ldots<\Phi_{3}(s)<1.99674 \ldots
$$

and that the distance between the points of maximum and of minimum for this function is quite close to $1 / 2$. This seems paradoxical, but the explanation is that $\Phi_{3}(s)$ is approximated well by a constant and perfectly well by a trigonometric polynomial of degree 1. 
Since $\Phi_{q}(s)$ is a 1-periodic analytic function in a strip, on the real axis it expands in a rapidly convergent Fourier series,

$$
\Phi_{q}(s)=\sum_{-\infty}^{\infty} c_{n}(q) e^{-2 \pi i n s} .
$$

B. M. Makarov called our attention to the existence of simple explicit formulas for $c_{n}(q)$ (see (11)).

In order to deduce these formulas and then apply them (in this and the next section), we need classical integral identities that involve the Riemann $\zeta$-function (see, e.g., [2]). The starting point is Riemann's formula

$$
\zeta(\lambda)=\frac{1}{\Gamma(\lambda)} \int_{0}^{\infty} \frac{t^{\lambda-1}}{d t} e^{t}-1 \quad(\operatorname{Re} \lambda>1)
$$

which is a consequence of the expansion

$$
\left(e^{t}-1\right)^{-1}=\sum_{n=1}^{\infty} e^{-n t} .
$$

Riemann's formula readily implies the relation

$$
2 \Gamma(\lambda)\left(1-2^{-\lambda}\right) \zeta(\lambda)=\int_{0}^{\infty} \frac{t^{\lambda-1} d t}{\sinh t} \quad(\operatorname{Re} \lambda>1) .
$$

Theorem 4. The Fourier coefficients of $\Phi_{q}(s)$ are given by the relation

$$
c_{n}(q)=\frac{(q-1)(q-2)}{q \log 2} \cdot \Gamma\left(\alpha_{n}\right) \zeta\left(\alpha_{n}\right)
$$

where $\alpha_{n}=\alpha+2 \pi i n / \log 2$.

Proof. We use the notation introduced before. We have:

$$
\begin{aligned}
c_{n}(q) & =\int_{0}^{1} H_{q}\left(2^{s}\right) e^{2 \pi i n s} d s \\
& =\sum_{-\infty}^{\infty} \int_{0}^{1} f_{q}\left(2^{k+s}\right) e^{2 \pi i n s} d s \\
& =\frac{1}{\log 2} \sum_{-\infty}^{\infty} \int_{2^{k}}^{2^{k+1}} t^{-1} f_{q}(t) e^{2 \pi i n \log _{2} t} d t \\
& =\frac{1}{\log 2} \int_{0}^{\infty} t^{-\alpha} f_{q}(t) t^{\alpha_{n}-1} d t \\
& =\frac{q-2}{2 \log 2} \int_{0}^{\infty} \frac{t^{\alpha_{n}-1} d t}{\sinh t},
\end{aligned}
$$

and it remains to refer to (10).

It should be noted that the combination of Theorems 3 and 4 includes (and refines) Lemma 4.

The Stirling formula shows that

$$
\log _{2} c_{0}(q) \sim\left(\log _{2} q\right) \cdot\left(\log _{2} \log _{2} q\right)
$$

as $q \rightarrow \infty$. In this sense, the value $q=3$ is not "typical", because the $\Gamma$-factor is responsible for the growth, and $\Gamma(\alpha)<1$ precisely for $1<\alpha<2$. We are going to show that the quantity $c_{0}(q)$ strictly increases with $q$. 

tions:

We recall that for $\operatorname{Re} \lambda>1$ the Riemann $\eta$-function admits the following representa-

$$
\begin{aligned}
\eta(\lambda) & =\left(1-2^{1-\lambda}\right) \zeta(\lambda) \\
& =\sum_{n=1}^{\infty}(-1)^{n-1} n^{-\lambda} \\
& =\frac{1}{\Gamma(\lambda)} \int_{0}^{\infty} \frac{t^{\lambda-1} d t}{e^{t}+1} .
\end{aligned}
$$

The function $\eta(\lambda)$ extends to an entire function, and the integral and the series in (12) still converge for $\operatorname{Re} \lambda>0$.

Clearly,

$$
c_{0}(q) \log 2=\left(2^{\alpha}-1\right) \Gamma(\alpha) \eta(\alpha) .
$$

Apparently, the following lemma is well known.

Lemma 5. We have $\eta^{\prime}(\lambda)>0$ for $\lambda \geq 1$.

Proof. Clearly, the sequence $\left\{n^{-\lambda} \log n \mid n \geq 2\right\}$ is monotone if $\lambda \geq \log _{2} e$, and in this case the claim is obvious. For other $\lambda \geq 1$, an "irregularity" occurs only under the passage from the first term to the second. So, it suffices to check the inequality

$$
\frac{\log 2}{2^{\lambda}}-\frac{\log 3}{3^{\lambda}}+\frac{\log 4}{4^{\lambda}}-\frac{\log 5}{5^{\lambda}}>0 .
$$

This is equivalent to the inequality

$$
1-a t^{a-1}+2 t-b t^{b-1}>0 \quad(0<t \leq 1 / 2),
$$

where $a=\log _{2} 3, b=\log _{2} 5$. For $t=1 / 2$ the latter inequality is true indeed (because $3^{5} \cdot 5^{3}<2^{15}$ ). Therefore, it suffices to show that the derivative in $t$ is negative, which is equivalent to the inequality

$$
a(a-1) t^{a-2}+b(b-1) t^{b-2}>2 .
$$

But the product of the two terms on the left is strictly greater than 1 for $0<t<1$ because $a+b<4$ and $a b(a-1)(b-1)>1$.

It is well known that $\left(d^{2} / d \lambda^{2}\right) \log \Gamma(\lambda)>0$ for $\lambda>0$, i.e., the function $\log \Gamma(\lambda)$ is strictly convex for $\lambda>0$. For clarity, we present a simple and general fact that implies this claim.

Let $\{X, \nu\}$ be a space with a nontrivial measure $\nu$, and let $f: X \rightarrow \mathbb{R}$ be a nonnegative function such that $f \in L^{1}(\nu)$. Then the function

$$
w(\lambda)=\int_{X} f(x)^{\lambda} \nu(d x)
$$

is well defined and analytic in the strip $0<\operatorname{Re} \lambda<1$. The next lemma is an easy consequence of the Cauchy inequality.

Lemma 6. If $0<\lambda<1$, then $w^{\prime}(\lambda)^{2} \leq w(\lambda) \cdot w^{\prime \prime}(\lambda)$.

Proof. Indeed,

$$
\begin{aligned}
w^{\prime}(\lambda)^{2} & =\left(\int_{X} f^{\lambda / 2} \cdot f^{\lambda / 2} \log f \nu(d x)\right)^{2} \\
& \leq \int_{X} f^{\lambda} \nu(d x) \cdot \int_{X} f^{\lambda} \log ^{2} f \nu(d x) \\
& =w(\lambda) \cdot w^{\prime \prime}(\lambda),
\end{aligned}
$$

and it is easy to see when the inequality is strict. 
Recall that $\Gamma^{\prime}(1)=-\gamma$, where $\gamma$ is the Euler constant. It is useful to keep in mind that

$$
\gamma=0.5772 \ldots<\log 2=0.6931 \ldots
$$

If the logarithm of a function is convex, then so is the function itself. Since $\Gamma(1)=$ $\Gamma(2)=1$, we see that the only positive zero of $\Gamma^{\prime}$ lies on $(1,2)$.

Theorem 5. The coefficient $c_{0}(q)$ is strictly monotone increasing with $q$.

Proof. By Lemma 5 , all the three functions $\Gamma(\lambda+1),\left(2^{\lambda}-1\right) / \lambda$, and $\eta(\lambda)$ are strictly monotone increasing as $\lambda \geq 1$.

Remark. We give another proof of Theorem 5, which employs Lemma 6 in a different way. Put $v(\lambda)=\eta(\lambda) \Gamma(\lambda)$. It is known that the Laurent series for $\zeta(\lambda)$ at $\lambda_{0}=1$ is of the following form:

$$
\zeta(\lambda)=\frac{1}{\lambda-1}+\gamma+\gamma_{1}(\lambda-1)+\cdots
$$

It easily follows that 1

$$
\eta^{\prime}(1)=(\gamma-(1 / 2) \log 2) \log 2 .
$$

Combined with the formula $\Gamma^{\prime}(1)=-\gamma$, this yields $v^{\prime}(1) / v(1)=-(1 / 2) \log 2$. Next, by Lemma $6,\left(d^{2} / d \lambda^{2}\right) \log v(\lambda)>0$, and the proof finishes readily.

Perhaps Theorem 5 by itself does not deserve the attention paid to it here. However, the point is that the function $c_{0}(q)$ admits analytic continuation from the semiaxis $q>2$, and though no Cantor functions correspond to "extraneous" $q$, it would be interesting to learn how $c_{0}(q)$ (along with some related functions) behaves for such $q$, i.e., for "imaginary" (as N. I. Lobachevsky would have said) Cantor functions. The proof given above allows us to show that monotonicity persists if we move a little to the left from the point $q=2$.

Since the $\Gamma$-function decays rapidly on the imaginary axis, the coefficients $c_{n}(q)$ with $n \neq 0$ are small compared to $c_{0}(q)$. The difference is particularly transparent when $q$ is not too large.

Since $|\zeta(\alpha+i t)| \leq \zeta(\alpha)$ for $t \in \mathbb{R}$, Theorem 4 yields the inequality

$$
\left|c_{n}(q)\right| / c_{0}(q) \leq\left|\Gamma\left(\alpha_{n}\right)\right| / \Gamma(\alpha) .
$$

It is known that $|\Gamma(1 / 2+i t)|^{2}=\pi / \cosh \pi t$ for $t \in \mathbb{R}$. This gives a clear idea of how small are, for instance, the numbers $\left|c_{n}(2 \sqrt{2})\right|$ for $n \neq 0$.

\section{§6. Analytic continuation of the function $I_{q}(\lambda)$}

The integral defining $I_{q}(\lambda)$ converges for $\operatorname{Re} \lambda>-\alpha$ and represents an analytic function in this half-plane. The right-hand side of (1) represents an entire function. Therefore, $I_{q}(\lambda)$ extends to a meromorphic function with singularities at $\lambda=-\alpha_{n}$, where (as before) $\alpha_{n}=\alpha+2 \pi i n / \log 2, n \in \mathbb{Z}$. These singularities are simple poles at the worst, and we shall indicate the residues.

Lemma 7. For $0<\operatorname{Re} \lambda<\alpha$, we have

$$
I_{q}(-\lambda)=\frac{1}{\Gamma(\lambda)} \int_{0}^{\infty} t^{\lambda-1} E_{q}(-t) d t .
$$

\footnotetext{
${ }^{1}$ In the popular handbook [5], the Euler constant is denoted by $\mathbf{C}$ and occurs in many formulas. However, the strange value $0.15986 \ldots$ is exhibited for $\eta^{\prime}(1)$ (item 1 in 4.325). Surely, these are the first decimal digits of the combination we give here.
} 
Proof. We represent the integral on the right as a double integral and change the order of integration:

$$
\begin{aligned}
\int_{0}^{\infty} & t^{\lambda-1} E_{q}(-t) d t=\int_{0}^{\infty} t^{\lambda-1}\left\{\int_{0}^{\infty} e^{-t x} d m_{q}(x)\right\} d t \\
& =\int_{0}^{\infty}\left\{\int_{0}^{\infty} t^{\lambda-1} e^{-x t} d t\right\} d m_{q}(x) \\
& =\Gamma(\lambda) \int_{0}^{\infty} \frac{d m_{q}(x)}{x^{\lambda}}=\Gamma(\lambda) I_{q}(-\lambda) .
\end{aligned}
$$

The following lemma can be deduced from the Wiener Tauberian theorem (see, e.g., [6. Chapter 9] about the latter), but we prefer to give a direct proof.

Lemma 8. Let $f \in L^{\infty}\left(\mathbb{R}_{+}\right)$. Suppose that the following mean value exists:

$$
A(f) \stackrel{\text { def }}{=} \lim _{T \rightarrow \infty} \frac{1}{T} \int_{0}^{T} f(t) d t .
$$

Then for $\varepsilon>0$ we have

$$
\lim _{\varepsilon \rightarrow 0} \int_{0}^{\infty} f(t / \varepsilon) \varphi(t) d t=A(f) \int_{0}^{\infty} \varphi(t) d t
$$

for every function $\varphi \in L^{1}\left(\mathbb{R}_{+}\right)$(and the limit exists).

Proof. If $f=$ const, the statement is trivial. This allows us to assume that $A(f)=0$. Denote by $K$ the collection of all $\varphi \in L^{1}\left(\mathbb{R}_{+}\right)$such that $\lim _{\varepsilon \rightarrow 0} \int_{0}^{\infty} f(t / \varepsilon) \varphi(t) d t=0$. Clearly, $K$ is a closed linear subspace of $L^{1}\left(\mathbb{R}_{+}\right)$, and we must show that $K=L^{1}\left(\mathbb{R}_{+}\right)$. By assumption, it easily follows that $K$ contains the indicators of all intervals $[0, b)$. Therefore, $K$ contains the indicators of all intervals $[a, b)$, and the linear combinations of the latter functions form a dense subset of $L^{1}\left(\mathbb{R}_{+}\right)$.

We need quite a particular case of Lemma 8, namely, the case where $\varphi(t)=e^{-c t}$, $c>0$, and $f(t)=f_{0}(t)+f_{1}(t)$, where $f_{0}$ is a (bounded, measurable) 1-periodic function and $f_{1}(t) \rightarrow 0$ as $t \rightarrow+\infty$. Then the claim of the lemma can be written in the form

$$
\lim _{\varepsilon \rightarrow 0} \varepsilon \int_{0}^{\infty} e^{-\varepsilon c t} f(t) d t=c^{-1} \int_{0}^{1} f_{0}(t) d t .
$$

In what follows, we use the notation of $\S 5$.

Theorem 6. The residues of the function $I_{q}(\lambda)$ are given by the formula

$$
\underset{\lambda=-\alpha_{n}}{\operatorname{Res}} I_{q}(\lambda)=(q-1)(q-2) \zeta\left(\alpha_{n}\right) / q \log 2 .
$$

In other words,

$$
\underset{\lambda=-\alpha_{n}}{\operatorname{Res}} I_{q}(\lambda)=c_{n}(q) / \Gamma\left(\alpha_{n}\right),
$$

where the $c_{n}(q)$ are the Fourier coefficients of $\Phi_{q}(s)$.

Remark. The fact that the representations (14) and (15) coincide follows from (11). Initially, our proof of (14) involved formula (10), Lemma 7, and some other (fairly long and artificial) arguments. In that context, the above coincidence seemed to be accidental. However, such phenomena almost never occur accidentally. Here we give the proof of (15) that leans upon Lemma 8 and appears to be fairly natural. 
Proof of Theorem 6. Below we assume that $0<\varepsilon<\alpha$. Since

$$
\underset{\lambda=-\alpha_{n}}{\operatorname{Res}} I_{q}(\lambda)=\lim _{\varepsilon \rightarrow 0} \varepsilon I_{q}\left(-\alpha_{n}+\varepsilon\right),
$$

by Lemma 7 we obtain

$$
\Gamma\left(\alpha_{n}\right) \underset{\lambda=-\alpha_{n}}{\operatorname{Res}} I_{q}(\lambda)=\lim _{\varepsilon \rightarrow 0} \varepsilon \int_{0}^{\infty} t^{\alpha_{n}-1-\varepsilon} E_{q}(-t) d t .
$$

The limit on the right in (16) does not change if the integration domain is reduced to $[1, \infty)$. Therefore,

$$
\begin{aligned}
\Gamma\left(\alpha_{n}\right) & \operatorname{Res}_{\lambda=-\alpha_{n}} I_{q}(\lambda)=\lim _{\varepsilon \rightarrow 0} \varepsilon \int_{1}^{\infty} t^{\alpha_{n}-1-\varepsilon} E_{q}(-t) d t & & \text { (by Lemma 7) } \\
& =\lim _{\varepsilon \rightarrow 0} \varepsilon \int_{1}^{\infty} t^{-1-\varepsilon} e^{2 \pi i n \log _{2} t}\left(H_{q}(t)-R_{q}(t)\right) d t & & \text { (by (9)) } \\
& =\frac{1}{\log 2} \lim _{\varepsilon \rightarrow 0} \varepsilon \int_{0}^{\infty} e^{-\varepsilon t \log 2} e^{2 \pi i n t}\left(\Phi_{q}(t)-R_{q}\left(2^{t}\right)\right) d t & & \text { (the substitution } \left.t \mapsto 2^{t}\right) \\
& =\int_{0}^{1} e^{2 \pi i n t} \Phi_{q}(t) d t & & \text { (by (13)) } \\
& =c_{n}(q) . & & \text { (by definition). }
\end{aligned}
$$

It has already been noted that, in a sense, the absence of the limit of $\lambda^{\alpha} I_{q}(\lambda)$ and $\lambda^{\alpha} E_{q}(-\lambda)$ as $\lambda \rightarrow+\infty$ is equivalent to the relation $\Phi_{q}(\lambda) \neq$ const. With some reservations, Theorem 6 allows us to add that this is also equivalent to the fact that $I_{q}(\lambda)$ does not extend to an entire function.

Together with (1), Theorem 6 enables us to evaluate some other integrals that involve Cantor functions. For example, if $q=4$, then $\alpha=2$, and we see that

$$
\int_{0}^{1} \frac{d t}{\left(1+C_{4}(t)\right)^{2}}=-2+\pi^{2} / 4
$$

Remark. In connection with a general formula due to Müntz that involves the $\zeta$-function, Titchmarsh (see [7]) gave the following formal relation involving an arbitrary function $F$ :

$$
\int_{0}^{\infty} x^{\lambda-1} \sum_{n=1}^{\infty} F(n x) d x=\zeta(\lambda) \int_{0}^{\infty} x^{\lambda-1} F(x) d x .
$$

Under minimal regularity assumptions, this formal relation can be justified. The Riemann formula ([7, Chapter $9, \S 8]$ ) arises if we put for $F(x)=e^{-x}$. It should be mentioned that this fact can be linked to the Möbius inversion formula.

In the same sense, our Lemma 7 corresponds to the following formal relation with an arbitrary distribution $g$ :

$$
\int_{0}^{\infty} x^{\lambda-1}\left\{\int_{0}^{\infty} e^{-x t} d g(t)\right\} d x=\Gamma(\lambda) \int_{0}^{\infty} x^{-\lambda} d g(x) .
$$

Taking $g=m_{q}$, we obtain Lemma 7. If $g$ corresponds to the counting measure on the natural numbers, we obtain the Riemann formula. Putting $g(t)=1-e^{-t}$, we obtain a standard integral on the left; it is calculated with the help of the residue theorem, and as a result we obtain the classical identity $\Gamma(\lambda) \Gamma(1-\lambda)=\pi / \sin \pi \lambda$.

The formula

$$
\int_{0}^{\infty} x^{\lambda-1}\left\{\int_{0}^{\infty} F(x t) d g(t)\right\} d x=\int_{0}^{\infty} x^{\lambda-1} F(x) d x \cdot \int_{0}^{\infty} t^{-\lambda} d g(t)
$$


combines the preceding ones. The invariant meaning of this formula is the following. Let $X$ be a unimodular locally compact group (i.e., a group with a doubly invariant Haar measure $d x$ ), and $\chi: X \rightarrow \mathbb{C}^{*}$ a continuous homomorphism. Then (as before, we neglect certain subtleties)

$$
\int_{X} \chi(x)\left\{\int_{X} F(x t) \nu(d t)\right\} d x=\int_{X} \chi(x) F(x) d x \cdot \int_{X} \chi(t)^{-1} \nu(d t) .
$$

We observe that this formula reduces to the preceding one if $X$ is the multiplicative group of positive numbers.

\section{$\S 7$. REAL ZEROS OF THE FUnCTION $E_{q}(i \lambda)$}

Formula (2) implies the relation

$$
e^{-i \lambda} E_{q}(2 i \lambda)=(1-\theta) f(\lambda, \theta)
$$

where $\theta=2 / q$ and

$$
\begin{aligned}
f(\lambda, \theta) & =\sum_{n=0}^{\infty} \theta^{n}(\sin \lambda) / 2^{n}\left(\sin \lambda / 2^{n}\right) \\
& =1+\theta(\cos \lambda / 2)+\theta^{2}(\cos \lambda / 2)(\cos \lambda / 4)+\cdots .
\end{aligned}
$$

This readily shows that $f(\lambda, \theta) \neq 0$ for $\lambda \in \mathbb{R}$ if $\theta<1 / 2$. Thus, for $q>4$ the function $E_{q}(i \lambda)$ has no real zeros.

On the other hand,

$$
(1-\theta) f(\lambda, \theta) \rightarrow e^{-i \lambda} E_{2}(2 i \lambda)=\lambda^{-1} \sin \lambda
$$

uniformly on each compact set, as $\theta \rightarrow 1$. So, if $\theta$ is sufficiently close to 1 (i.e., $q$ is sufficiently close to 2$)$, then $f(\lambda, \theta)$ takes negative values on the interval $(\pi, 2 \pi)$. For fixed $\theta$, this function is almost periodic; therefore, it has infinitely many real zeros.

The following lemma is obvious.

Lemma 9. If $\pi<\lambda<2 \pi$ and $0<\theta<1$, then $\partial f / \partial \theta<0$.

Thus, as $\theta$ decreases (in other words, as $q$ increases), the graph of $f(\lambda, \theta)$ raises for every $\lambda \in(\pi, 2 \pi)$.

Since the sequence $\left\{f\left(\lambda / 2^{n}, \theta\right)\right\}$ is bounded, we observe that (17) is equivalent to the functional equation

$$
f(\lambda, \theta)=1+(\theta \cos \lambda / 2) f(\lambda / 2, \theta) .
$$

Lemma 10. For $\theta \in(0,1)$ fixed, if the inequality $f(\lambda, \theta) \leq 0$ is fulfilled for some $\lambda>2 \pi$, then the strict inequality $f(\lambda, \theta)<0$ is fulfilled for some $\lambda \in(\pi, 2 \pi)$.

This lemma can be given a nearly set-theoretic proof, which will be postponed till $\S 8$.

Theorem 7. There exists $q_{0}$ such that the function $E_{q}(i \lambda)$ has infinitely many real zeros for $q<q_{0}$ and has none for $q>q_{0}$.

This statement is an immediate consequence of the above lemmas. Furthermore, Lemma 10 allows us to easily "catch" the critical value $q_{0}$ with the use of a calculator. It turns out that $2.74<q_{0}<2.75$; in particular, the function $E_{3}(i \lambda)$ has no real zeros.

Now we present more information about the position and the dynamics of the zeros of $f(\lambda, \theta)$. In particular, we describe in detail what happens in the strip $-2 \pi \leq \operatorname{Re} \lambda \leq 2 \pi$ as the parameter $\theta$ varies.

First, we list the consequences of general considerations. We emphasize that $f$ is viewed as a function of $\lambda$, and $\theta \in(0,1)$ is regarded as a parameter. When talking of the 
"limit function", we mean $\lambda^{-1} \sin \lambda$. The distinction between the limit function and the functions before taking the limit turn out to be quite substantial.

We denote by $\Lambda=\Lambda(\theta)$ the set of all zeros of $f$. Since $f$ is real (on the real axis) and even, we see that $\Lambda$ is invariant under the mappings $\lambda \rightarrow-\lambda$ and $\lambda \rightarrow \bar{\lambda}$. There are no zeros on the imaginary axis.

The function $f$ belongs to the so-called Cartwright class, has exponential type 1, and has symmetric indicator diagram. Therefore, besides the inequality

$$
\sum_{\lambda \in \Lambda}|\lambda|^{-1-\varepsilon}<\infty \text { for all } \varepsilon>0,
$$

which is valid for all functions of exponential type, this function obeys the relations

$$
\sum_{\lambda \in \Lambda}\left|\operatorname{Im} \lambda^{-1}\right|<\infty \quad \text { and } \quad \sum_{\lambda \in \Lambda}\left|\lambda^{-1}\right|=\infty
$$

(see, e.g., [8, Lecture 17]), which tell us that the zeros of this function are placed quite regularly. We note that the first of the conditions (19) is close to the Blaschke condition and is proved readily, whereas the proof of the second requires rather subtle Levinson's theorem.

Conditions (19) mean that in each angle $|\operatorname{Im} \lambda| \leq \varepsilon|\operatorname{Re} \lambda|$ there are considerably more zeros than off this angle. This gives no information about the existence of zeros far away from the real axis, but in the case in question the classical Bohr theorem applies (see, e.g., [9] Chapter 6]). Indeed, $f$ is an entire almost-periodic function, and the precise bounds \pm 1 of the spectrum do not belong to the spectrum. By the Bohr theorem, this implies that the set $\{\operatorname{Im} \lambda \mid \lambda \in \Lambda\}$ is unbounded, and this is a point of distinction between the functions before the limit passage and the limit function.

For integers $k, r \geq 0$, we put $l=2^{r}(2 k+1)$. Then

$$
f(\pi l, \theta)=\left(1-2 \theta^{r}+\theta^{r+1}\right) /(1-\theta) .
$$

In particular, $f(\pi l, \theta)>0$. Let

$$
\Delta_{2 k+1}=((2 k+1) \pi, 2(k+1) \pi) .
$$

Clearly, $\left\{\Delta_{2 k+1}\right\}$ are precisely the intervals on the semiaxis $\lambda>0$, on which the limit function is negative. By the Rouché theorem, for $\theta$ close to 1 the $\varepsilon$-neighborhood of the segment $\cos \left(\Delta_{2 k+1}\right)$ contains two simple zeros of the function $f(\lambda, \theta)$. Since this function is positive at the endpoints of the segment and takes negative values on $\Delta_{2 k+1}$ (if $\theta$ is close to 1 ), we see that the two zeros belong to the interval $\Delta_{2 k+1}$.

We denote by $\theta_{k}$ the minimum value of $\theta$ for which $f(\lambda, \theta)$ has a zero on the interval $\Delta_{2 k+1}$. By Lemma 10, $\theta_{k}>\theta_{0}$ for $k \geq 1$. At the same time, we have the following elementary fact.

\section{Theorem 8.}

$$
\varliminf_{k \rightarrow \infty} \theta_{k}=\theta_{0} \quad \text { and } \quad \varlimsup_{k \rightarrow \infty} \theta_{k}=1 .
$$

Proof. Let $m \geq 2$. In accordance with (17), we put $f=f_{m}+g_{m}$, where $g_{m}$ is the sum of all terms of the series in (17) that involve $\theta^{n}$ with $n \geq m$. Then $f_{m}\left(\lambda+2^{m} \pi, \theta\right)=f_{m}(\lambda, \theta)$, and $\left|g_{m}(\lambda, \theta)\right| \leq \theta^{m} /(1-\theta)$ for all $\lambda$ and $\theta$. Let $\theta_{0}<\tau<1$. There exists $\lambda_{0} \in(\pi, 2 \pi)$ such that $f\left(\lambda_{0}, \tau\right)<0$. We put $\lambda_{m}=\lambda_{0}+2^{m} \pi$. Then $\lambda_{m} \in \Delta_{2^{m}+1}$ and

$$
f\left(\lambda_{m}, \tau\right) \leq f\left(\lambda_{0}, \tau\right)+2 \tau^{m} /(1-\tau)<0
$$

if $m$ is sufficiently large. Thus, $\theta_{2^{m}+1}<\tau$ for such $m$. This implies the first relation in (21). 

have

Let $\lambda \in \Delta_{2^{m}-1}$. Then $\cos \lambda / 2^{n}>0$ for $1 \leq n<m$. Therefore, by (17), for such $\lambda$ we

$$
f(\lambda, \theta)>1-\theta^{m} /(1-\theta) .
$$

Consequently, $f(\lambda, \theta)>0$ if $\theta<t_{m}$, where $t_{m}$ is the positive root of the equation $1-t-t^{m}=0$ (we observe that $t_{m}<1$ ). It follows that $\theta_{2^{m}-1}>t_{m}$. Since $t_{m} \rightarrow 1$ as $m \rightarrow \infty$, this implies the second relation in (21).

We note that $\Delta_{2^{m}-1}$ and $\Delta_{2^{m}+1}$ are neighboring intervals in the family $\left\{\Delta_{2 k+1}\right\}$ and, as we have shown, the zeros of $f$ have a tendency to stay at the intervals $\Delta_{2_{m}-1}$ and to quickly disappear from the intervals $\Delta_{2^{n}+1}$ as $q$ grows.

Lemma 11. If $t>0$ and $m \geq 1$, then $\operatorname{Im} f\left(2^{m} \pi+i t, \theta\right)>0$.

Proof. We use the first line in (17). For brevity, we put $\lambda=2^{m} \pi+i t$. The point is simply that the imaginary parts of all terms are nonnegative, and positive for the most of them. First, $\sin \lambda=i \sinh t$. Second,

$$
\operatorname{Re} \sin \left(\lambda / 2^{n}\right)= \begin{cases}0 & \text { if } n \leq m, \\ \left(\sin \pi / 2^{n-m}\right) \cdot\left(\cosh t / 2^{n}\right) & \text { if } n>m,\end{cases}
$$

and the proof is finished.

Lemma 11 and formula (20) imply the following statement.

Lemma 12. On the lines $\operatorname{Re} \lambda=2^{m} \pi, m=1,2, \ldots$, we have $f(\lambda, \theta) \neq 0$.

Clearly, $f(i t, \theta)>0$. Moreover, there are no zeros on the line $\operatorname{Re} \lambda=\pi$ : the same argument as in the proof of Lemma 11 shows that $\operatorname{Im} f(\pi+i t, \theta)<0$ for $t>0$. Obviously, there are no zeros also on the vertical lines symmetric to the lines indicated.

Lemma 13. Let $\delta>0$. Then in every strip $\{\lambda \in \mathbb{C}|| \operatorname{Re} \lambda \mid \leq R<\infty\}$ we have

$$
f(\lambda, \theta) \rightarrow \infty \quad \text { as } \lambda \rightarrow \infty,
$$

uniformly in $\theta \geq \delta$.

Proof. Suppose first that $R<\pi / 2$. Then, in essence, we use a positive definiteness argument. Clearly, $f$ has the representation

$$
f(\lambda, \theta)=\int_{-1}^{1} \cos \lambda x \nu(d x),
$$

where $\nu$ is a positive measure (of total mass $(1-\theta)^{-1}$ ). Therefore, if $\lambda=\xi+i \eta$, then

$$
|f(\lambda, \theta)| \geq \operatorname{Re} f(\lambda, \theta)=\int_{-1}^{1}(\cos \xi x)(\cosh \eta x) \nu(d x) \geq(\cos R) f(i \eta, \theta),
$$

and the proof is finished in the case in question. Thus, it suffices to show that if the claim is valid for some $R$, then it is valid also with $2 R$ in place of $R$. But this easily follows from the functional equation (18), because the function $\cos \lambda$ is bounded away from zero off every $\varepsilon$-neighborhood of the set of its zeros.

Theorem 9. Let $m$ be a natural number, and let $0<\theta<1$. Then, with multiplicity taken into account, the function $f(\lambda, \theta)$ has precisely $2^{m}$ zeros in the strip $\{\lambda \in \mathbb{C} \mid 0<$ $\left.\operatorname{Re} \lambda<2^{m} \pi\right\}$. 
Proof. By Lemma 12, there are no zeros on the boundary of the strip. Next, it suffices to prove the theorem under the additional assumption $\theta \geq \delta>0$. But if so, then, by Lemma 13, we can choose $T$ in such a way that all zeros lie strictly inside the rectangle $\left\{\lambda \in \mathbb{C}\left|0 \leq \operatorname{Re} \lambda \leq 2^{m} \pi,\right| \operatorname{Im} \lambda \mid \leq T\right\}$. Since for any $\theta$ in question there are no zeros on the boundary of the rectangle, the number of zeros does not depend on $\theta$. Consequently, there are precisely $2^{m}$ zeros, because precisely so many of them exist for $\theta$ close to 1 (this has already been mentioned: we deal with the zeros of the limit function $\lambda^{-1} \sin \lambda$ slightly displaced).

Corollary. For every $\theta \in(0,1)$ there are two zeros in the strip $\{\lambda \in \mathbb{C} \mid \pi<\operatorname{Re} \lambda<2 \pi\}$. For $\theta>\theta_{0}$ these zeros are real and distinct. For $\theta=\theta_{0}$, a real zero of multiplicity 2 arises. For $\theta<\theta_{0}$ there are two complex conjugate zeros, which "disappear at infinity" as $\theta \rightarrow 0$.

Remark. Since there are four zeros in the strip $0<\operatorname{Re} \lambda<4 \pi$, it follows that two of them are in the strip $\{\lambda \in \mathbb{C} \mid 2 \pi<\operatorname{Re} \lambda<4 \pi\}$. So, it can be expected that the picture is similar to the above (with $\theta_{0}$ in place of $\theta_{1}$ ); however, we have not managed to repeat the entire argument. Thus, it is even less clear how the zeros "exit" from other intervals $\Delta_{2 k+1}$; nor is it understood whether two different intervals may be "cleared" simultaneously (whether two different multiple zeros may occur for some $\theta$ ), whether multiple zeros may occur off the real line, etc. Some numerical simulation may be useful here. In particular, it would be interesting to look at a certain number of the first $\theta_{k}$ 's.

\section{§8. AN ABSTRACT VERSION OF THE LEMMA ON NEGATIVE VAlUES}

In this section we prove a simple and general theorem, which includes Lemma 10 (on the zeros of $f(\lambda, \theta)$ in $(\pi, 2 \pi))$ among other things.

Let $X$ be a nonempty set, let $A \subseteq X$, and let $\omega: X \rightarrow X$ be a mapping. We denote by $\omega_{0}$ the identity mapping and, for natural $n$, we put $\omega_{n}=\omega \circ \omega_{n-1}$. The set $\left\{\omega_{n}(x)\right\}$ is called the orbit of a point $x$. In order to lighten the final statements, the main relations among the objects introduced in what follows will be established beforehand. We observe that all the conditions (c1)-(c8) are fulfilled if $X=\mathbb{R}_{+}, \omega(x)=x / 2$, and $g(x)=\theta \cos (x / 2)$, where $0<\theta<1$. So, the two theorems proved below are applicable to equation (18).

We assume that the set $A$ is invariant and absorbing, that is,

$$
\omega(a) \in A \text { for all } a \in A,
$$

and for every $x \in X$ we have

$$
\omega_{n}(x) \in A \text { if } n \text { is sufficiently large. }
$$

The symbol $m_{A}(x)$ denotes the minimum $n$ for which (c2) is satisfied. Let $g: X \rightarrow \mathbb{R}$ be a function such that

$$
\begin{gathered}
\{g(x) \mid x \in X\}=\{g(a) \mid a \in A\}, \\
g(\omega(a)) \geq 0 \text { for all } a \in A,
\end{gathered}
$$

and

$$
g(\omega(x))=g(\omega(a)) \text { if } g(x)=g(a) \text { and } g(\omega(x))>0 \quad(x \in X, a \in A) .
$$

We shall consider the real solutions $f$ of the functional equation

$$
f(x)=1+g(x) f(\omega(x)) .
$$

Assuming that $X, A, \omega$, and $g$ are subject to the above conditions, we want to know whether it is true that $f(a)<0$ (or at least $f(a) \leq 0$ ) for some point $a \in A$ if the set 
$\{x \in X \mid f(x) \leq 0\}$ is nonempty. Generally speaking, the conditions listed above $d o$ not ensure the existence of a point $a \in A$ with $f(a) \leq 0$ (later we shall explain how to construct examples).

We fix a point $x_{0}$ in $X$ and put $x_{n}=\omega_{n}\left(x_{0}\right)$. From (Eq) we deduce that $f\left(x_{0}\right)=$ $1+g\left(x_{0}\right) f\left(x_{1}\right)$ and for all integers $n>0$ we have

$$
f\left(x_{0}\right)=1+h_{0}\left(x_{0}\right)+h_{1}\left(x_{0}\right)+\cdots+h_{n-1}\left(x_{0}\right)+h_{n}\left(x_{0}\right) f\left(x_{n+1}\right),
$$

where

$$
h_{k}\left(x_{0}\right)=g\left(x_{0}\right) \cdot g\left(x_{1}\right) \cdots g\left(x_{k}\right) .
$$

The next lemma involves nothing except the definitions.

Lemma 14. Suppose that $g\left(x_{k}\right) \geq 0$ and $f\left(x_{k}\right) \geq 0$ for all $k$ sufficiently large. Then

$$
f\left(x_{0}\right)=1+\sum_{k=0}^{\infty} h_{k}\left(x_{0}\right)+\lim _{n \rightarrow \infty} h_{n}\left(x_{0}\right) f\left(x_{n+1}\right),
$$

the series converges absolutely, and the limit exists.

Proof. If $n$ is sufficiently large, then in (22) either all but finitely many terms are nonnegative, or all but finitely many terms are nonpositive.

In what follows, we assume throughout that $f$ is a solution of (Eq) and conditions (c1)(c5) are fulfilled. These assumptions are not even repeated in all statements. Naturally, in examples a particular assumption may or may not be fulfilled.

By (c3), for a given $x_{0}$ we can find $a_{0} \in A$ with $g\left(x_{0}\right)=g\left(a_{0}\right)$.

Lemma 15. Suppose that $f\left(x_{0}\right) \leq 0$ and $f\left(x_{k}\right) \geq 0$ for $k \geq 1$. If $f\left(a_{k}\right)>0$ for all $k$, then $g\left(x_{k}\right)=g\left(a_{k}\right)>0$ for $k \geq 1$.

Proof. Since $f\left(x_{0}\right) \leq 0$ and $f\left(x_{1}\right) \geq 0$, from (Eq) we deduce that $g\left(x_{0}\right)<0$. If $g\left(x_{1}\right) \leq 0$, then

$$
0 \geq f\left(x_{0}\right)=1+g\left(x_{0}\right)+g\left(x_{0}\right) g\left(x_{1}\right) f\left(x_{2}\right) \geq 1+g\left(x_{0}\right)=1+g\left(a_{0}\right) .
$$

Invoking (c4), we obtain

$$
0<f\left(a_{0}\right)=1+g\left(a_{0}\right)+g\left(a_{0}\right) g\left(a_{1}\right) f\left(a_{2}\right) \leq 1+g\left(a_{0}\right) \leq 0,
$$

a contradiction. Thus, $g\left(x_{1}\right)>0$. Condition (c5) implies that $g\left(x_{1}\right)=g\left(a_{1}\right)$. It remains to proceed by induction on $k$, the passage from $k$ to $k+1$ is done similarly.

Theorem 10. Suppose $f$ is a bounded function. If $f(x) \leq 0$ for at least one $x \in X$, then $f(a) \leq 0$ for some $a \in A$.

Proof. We suppose that $f(a)>0$ for all $a \in A$, and arrive at a contradiction. Put

$$
l=\min \left\{m_{A}(x) \mid f(x) \leq 0\right\}
$$

and let $m_{A}\left(x_{0}\right)=l$. Then $f\left(x_{0}\right) \leq 0$ and $f\left(x_{k}\right) \geq 0$ for $k \geq 1$. Indeed, if $k \geq l$, then $x_{k} \in A$, and if $1 \leq k<l$, the claim follows from the minimality of $l$. By (c3), there is $a_{0} \in A$ with $g\left(x_{0}\right)=g\left(a_{0}\right)$. Lemma 15 implies that $g\left(x_{k}\right)=g\left(a_{k}\right)$ for all $k$. Since $f$ is bounded, Lemma 14 shows that $f\left(x_{0}\right)=f\left(a_{0}\right)$ because $f\left(a_{0}\right) \leq 0$.

Theorem 10 yields a weak version of Lemma 10: if the function $f(\lambda, \theta)$ has at least one positive zero, then it has a zero on the interval $(\pi, 2 \pi)$, and this statement suffices for the proof of Theorem 7. Below we shall present an example showing that the boundedness assumption is essential in Theorem 10. However, in the next theorem (which contains Lemma 10 entirely) the boundedness condition is immaterial. Yet, in Theorem 10 instead of boundedness we may assume condition (c8) stated below; the same proof applies, and moreover, Lemma 14 is not needed. 
Theorem 11. Suppose that, besides (c1)-(c5), the following conditions are fulfilled:

$$
\begin{gathered}
g(x) \neq-1 \quad \text { for all } x \in X, \\
g\left(\omega_{2}(a)\right)>0 \text { for all } a \in A, \\
\text { if } g\left(x_{k}\right)=g\left(a_{k}\right) \text { for all } k \text {, then } x=a .
\end{gathered}
$$

In this case, if $f(x) \leq 0$ for some $x \in X \backslash A$, then $f(a)<0$ for some $a \in A$.

Proof. In essence, we follow the same outline. Suppose that $f(a) \geq 0$ for all $a \in A$. We redefine $l$ as follows:

$$
l=\min \left\{m_{A}(x) \mid f(x) \leq 0, x \notin A\right\} .
$$

Observe that now $l \geq 1$. Take $x_{0} \in X \backslash A$ with $m_{A}\left(x_{0}\right)=l$. Then $f\left(x_{0}\right) \leq 0$ and $f\left(x_{k}\right) \geq 0$ for $k \geq 1$. There exists $a_{0} \in A$ with $g\left(x_{0}\right)=g\left(a_{0}\right)$.

If $g\left(x_{0}\right)>0$, then $f\left(x_{1}\right)<0$. Thus, we may assume that $g\left(x_{0}\right)<0$.

Suppose that $g\left(x_{1}\right) \leq 0$. Then the inequality

$$
0 \geq f\left(x_{0}\right)=1+g\left(x_{0}\right)+g\left(x_{0}\right) g\left(x_{1}\right) f\left(x_{2}\right)
$$

implies $1+g\left(x_{0}\right) \leq 0$. By $(\mathrm{c} 6)$, the inequality is strict, that is, $1+g\left(a_{0}\right)<0$, and we arrive at a contradiction:

$$
0 \leq f\left(a_{0}\right)=1+g\left(a_{0}\right)+g\left(a_{0}\right) g\left(a_{1}\right) f\left(a_{2}\right)<0
$$

because $g\left(a_{1}\right) \geq 0$ by (c4). Therefore, $g\left(x_{1}\right)>0$ and $g\left(x_{1}\right)=g\left(a_{1}\right)$.

The relation $g\left(x_{k}\right)=g\left(a_{k}\right)>0$ for $k \geq 2$ is proved by induction. The induction starts in fact (i.e., in the sense given to this notion by the ancients who meant the possibility of almost literal repetition of the arguments under the passage from 2 to 3 , from 3 to 4 , etc.) with $k=2$, and we analyze this case in detail. Suppose that $g\left(x_{2}\right) \leq 0$. Then

$$
\begin{aligned}
0 & \geq f\left(x_{0}\right)=1+g\left(x_{0}\right)+g\left(x_{0}\right) g\left(x_{1}\right)+g\left(x_{0}\right) g\left(x_{1}\right) g\left(x_{2}\right) f\left(x_{3}\right) \\
& \geq 1+g\left(x_{0}\right)+g\left(x_{0}\right) g\left(x_{1}\right),
\end{aligned}
$$

so that

$$
\begin{aligned}
0 & \leq f\left(a_{0}\right)=1+g\left(x_{0}\right)+g\left(x_{0}\right) g\left(x_{1}\right)+g\left(x_{0}\right) g\left(x_{1}\right) g\left(a_{2}\right) f\left(a_{3}\right) \leq g\left(x_{0}\right) g\left(x_{1}\right) g\left(a_{2}\right) f\left(a_{3}\right) \\
& \leq 0,
\end{aligned}
$$

whence $f\left(a_{3}\right)=0$. Indeed, $g\left(x_{0}\right)<0, g\left(x_{1}\right)>0$, and $g\left(a_{2}\right)>0$ by $(\mathrm{c} 7)$. But $f\left(a_{3}\right)=$ $1+g\left(a_{3}\right) f\left(a_{4}\right)$, consequently, $f\left(a_{4}\right)<0$.

Now it can be said that the passage from $k-1$ to $k$ is done similarly. But if $g\left(x_{k}\right)=$ $g\left(a_{k}\right)$ for all $k$, then $x_{0}=a_{0}$ by (c8), and we arrive at a contradiction because $x_{0} \notin A$.

Remark. Of course, the assumptions can vary. The argument "by contradiction" shortens the way to the result, but it can be converted to a constructive argument, which, given $l$, provides several points (among the $x_{k}$ and $a_{k}$ ) such that $f$ is negative at some of them. In specific situations (like Lemma 10) such a proof may happen to be much more useful.

Construction of examples. In our arguments, at most two orbits were involved in each case. Therefore, we can apply what is called "retrograde analysis" by chess players. We agree that $X$ and $\omega$ constitute the following scheme:

$$
\left\{\begin{array}{l}
x_{0} \rightarrow x_{1} \rightarrow x_{2} \rightarrow \cdots, \\
a_{0} \rightarrow a_{1} \rightarrow a_{2} \rightarrow \cdots,
\end{array}\right.
$$

in which all points are pairwise distinct, and the arrows symbolize the action of $\omega$. We restrict ourselves to constructing examples that will show that the boundedness assumption in Theorem 10 and condition (c8) in Theorem 11 cannot be lifted. Moreover, it can be assumed that $A=X \backslash\left\{x_{0}\right\}$. 
Formula (22) implies that on the orbit $\left\{x_{n}\right\}$ all values $f\left(x_{n}\right)$ are uniquely determined by $f\left(x_{0}\right)$ if $g\left(x_{n}\right) \neq 0$ for all $n$.

We fix positive numbers $\alpha$ and $\beta$ with $\alpha+\beta<1$ and put

$$
g\left(x_{0}\right)=g\left(a_{0}\right)=-\alpha \quad \text { and } \quad g\left(x_{k}\right)=g\left(a_{k}\right)=\beta \text { for } k \geq 1 .
$$

It is easily seen that all conditions except (c8) are fulfilled and

$$
\alpha \beta^{k} f\left(x_{k+1}\right)=-f\left(x_{0}\right)+\left(1-\alpha-\beta+\alpha \beta^{k}\right) /(1-\beta) .
$$

Therefore, if $\left|f\left(x_{0}\right)\right| \leq(1-\alpha-\beta) /(1-\beta)$, then $f\left(x_{k}\right)>0$ for $k \geq 1$, and all this remains valid after replacement of $\left\{x_{n}\right\}$ by $\left\{a_{n}\right\}$. We note that the sequence $\left\{f\left(x_{n}\right)\right\}$ is bounded if and only if $f\left(x_{0}\right)=(1-\alpha-\beta) /(1-\beta)$.

In particular, if we put $f\left(x_{0}\right)=0, f\left(a_{0}\right)=(1-\alpha-\beta) /(1-\beta)$, we obtain an example showing that the boundedness condition in Theorem 10 cannot be dropped, and if we put $f\left(x_{0}\right)=-(1-\alpha-\beta) /(1-\beta), f\left(a_{0}\right)>0$, we obtain an example in which all conditions of Theorem 11 are satisfied except (c8) and, moreover, $f\left(x_{0}\right)<0$, but $f(x)>0$ for $x \neq x_{0}$.

\section{REFERENCES}

[1] Victor H. Moll, The evaluation of integrals: a personal story, Notices Amer. Math. Soc. 49 (2002), no. 3, 311-317. MR 2002m:11105

[2] A. Erdélyi, W. Magnus, F. Oberhettinger, and F. Tricomi, Higher transcendental functions. Vol. I, McGraw-Hill, New York, etc., 1953. MR 15:419i

[3] E. Seneta, Regularly varying functions, Lecture Notes in Math., vol. 508, Springer-Verlag, BerlinNew York, 1976. MR 56:12189

[4] N. M. Korobov, Trigonometric sums and their applications, "Nauka", Moscow, 1989; English transl., Exponential sums and their applications, Math. Appl. (Soviet Ser.), vol. 80, Kluwer Acad. Publishers Group, Dordrecht, 1992. MR 93a:11068

[5] I. S. Gradshtein and I. M. Ryzhik, Table of integrals, sums, series, and products, 4th ed., Fizmatgiz, Moscow, 1962; English transl., Academic Press, New York-London, 1965. MR 28:5198]

[6] W. Rudin, Functional analysis, McGraw-Hill, New York, etc., 1973. MR 51:1315]

[7] E. C. Titchmarsh, The theory of the Riemann zeta-function, Clarendon Press, Oxford, 1951. MR 13:741c

[8] B. Ya. Levin, Lectures on entire functions, Transl. Math. Monogr., vol. 150, Amer. Math. Soc., Providence, RI, 1996. MR 97j:30001

[9] Distribution of zeros of entire functions, Gostekhizdat, Moscow, 1956; English transl., Amer. Math. Soc., Providence, RI, 1964. MR 28:217

Mathematics Department, Moscow State Pedagogical University, Moscow, Russia

E-mail address: evgeny.gorin@mtu-net.ru

Mathematics Department, Moscow State Pedagogical University, Moscow, Russia

Received 10/JUN/2002

Translated by S. V. KISLYAKOV 